\title{
Fiber Laser Sensing System and Its Applications
}

\author{
Yuliang LIU, Wentao ZHANG, Tuanwei XU, Jun HE, Faxiang ZHANG, and Fang LI \\ Optoelectronic System Laboratory, Institute of Semiconductors, Chinese Academy of Sciences, Beijing, 100083, China \\ *Corresponding author: Yuliang LIU_ E-mail: ylliu@semi.ac.cn
}

\begin{abstract}
An overview of fiber laser sensing is presented. The design and the characteristics of distributed feedback (DFB) fiber lasers for high performance sensing applications are described. Demodulation techniques based on unbalanced fiber interferometer are discussed, especially for the noise level, the dynamic range, and the crosstalk in dense-wavelength-division multiplexing. Finally, the fiber laser sensing system configurations and field demonstrations for different applications are illustrated.
\end{abstract}

Keywords: DFB, fiber laser, sensor, hydrophone, accelerometer

\section{Introduction}

Fiber sensor technologies have attracted great interests during the past few decades due to the advantages mostly discussed as high sensitivity, electromagnetic interference (EMI) immunity, low cost, electrically passive, conveniently multiplexing, and high reliability [1-2]. Rapid technology advances of telecommunication industry accelerate the development of fiber sensing. A considerable improvement in laser component was achieved by use of distributed feedback (DFB) fiber laser (FL) structure, which has permitted high performance to be obtained from fiber-optic interferometric sensors interrogated by FL sources [3]. DFB fiber lasers can also be used as sensor elements on their own [4], which provide new possibilities for developing high-performance sensors with reduced size and complexity. They are capable of resolving length changes in optical fiber around three orders of magnitude smaller than that achieved with conventional, remotely interrogated fiber-optic interferometers [3] and provide an inherent wavelength multiplexing capability [5].
This paper reviews the technology that has been developed for high performance fiber laser sensing systems. We start with a review of the realization of high performance DFB fiber lasers in Section 2. High resolution interrogation schemes for sensing systems based on DFB fiber laser is discussed in Section 3. Section 4 covers the fiber laser transducers for different applications and the field demonstrations of FL sensing systems.

\section{DFB fiber lasers for sensing applications}

\subsection{Fabrication of single mode fiber lasers}

Fiber laser consists of a length of $\mathrm{Er}^{3+}$-doped or $\mathrm{Yb}^{3+} / \mathrm{Er}^{3+}$-codoped fiber with two reflecting mirrors formed at each end, and is referred to as distributed Bragg reflector (DBR) laser [6-7]. In order to operate in single mode, a short cavity with narrow-bandwidth fiber Bragg grating(FBG) is required to effectively limit the spectrum therein allowing only a single dominant mode to be supported. The longitudinal mode spacing is given by

$$
\Delta v=c / 2 n l
$$

Received: 20 September 2010 / Revised version: 15 October 2010

(C) The Authors(s) 2010.This article is published with open access at Springerlink.com 
where $l$ is the effective length between the mirrors, $n$ is the effective reflective index of the fiber core, and $c$ is the light velocity in a vacuum. For a strong fiber grating with the reflection bandwidth of $0.2 \mathrm{~nm}$, the effective length should be less than $4 \mathrm{~mm}$ for single mode operation, which requires the length between FBGs to be much shorter [8]. Though stable performance has been achieved from these lasers in laboratory experiments, they are still susceptible to mode hopping if the temperature of gain section is not maintained constant.

To improve laser stability, DFB laser structure was used [9-11]. By introducing a $\pi$ phase shift, the grating resonance is moved to the center of the grating reflection band, which makes DFB fiber laser operate robustly in a single longitudinal mode [12].

The configuration of DFB fiber laser is shown in Fig. 1. A phase-shifted grating is formed into a length of $\mathrm{Er}^{3+}$ fiber, whose ends are spliced to a matching passive fiber for reducing splice loss. The grating is pumped with a $980 \mathrm{~nm}$ or $1480 \mathrm{~nm}$ semiconductor laser.

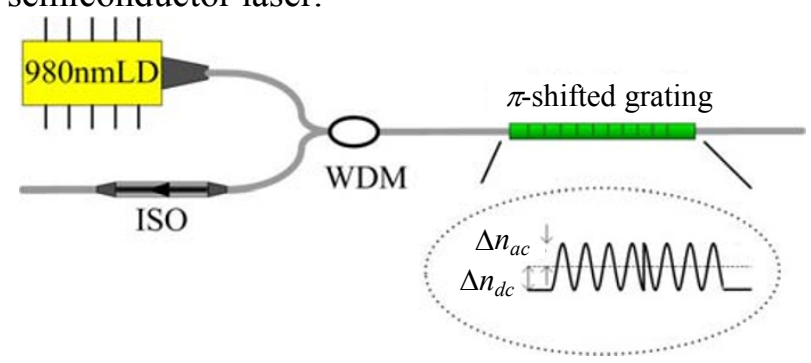

Fig. 1 DFB fiber laser configuration.

There are different techniques to fabricate FBGs in optical fiber [13-14]. The phase-mask method offers a high probability of mass production with good repeatability at low cost. To introduce a phase shift in the uniform period structure, ultraviolet (UV) post-processing[15] or occlusion processing is needed, and $\mathrm{Er}^{3+}$-doped fiber should exhibit high photosensitivity. Also, the active fiber should exhibit sufficient gain to meet with the lasing criteria by equaling the cavity power gain to total loss including background loss and power loss through the grating. The efficiency of DFB fiber laser can be improved by optimizing the parameters (e.g., grating strength, position of the phase shift, grating profile) [16-17].

\subsection{Line-width and noise characteristics of DFB FL}

DFB fiber lasers have been fabricated in our laboratory with $40 \mathrm{~mm}$ long. Unequal output power from each side is due to the non-centrally located phase shift. The left output power measured was about $100 \mu \mathrm{W}$ for $100 \mathrm{~mW}$ of $980 \mathrm{~nm}$ pump power, and the threshold pump power was about $9 \mathrm{~mW}$. The laser full-width half-maximum (FWHM) line-width was measured by using self-heterodyne method [18] with a $25 \mathrm{~km}$ long delay fiber and a fiber coupled acousto-optic modulator $(55 \mathrm{MHz})$. An optical self-heterodyne measurement is shown in Fig. 2. For the usual case of Lorentzian-shaped spectrum, the measured FWHM line-width was about $3 \mathrm{kHz}$.

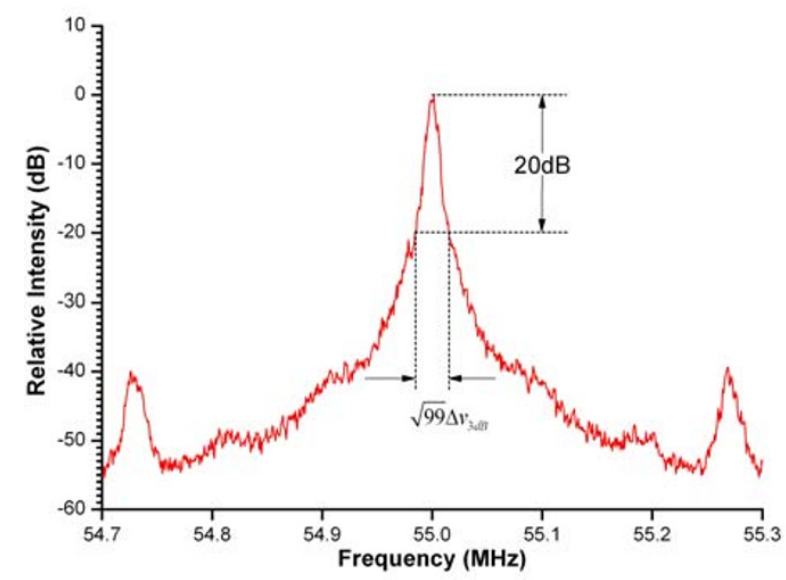

Fig. 2 DFB fiber laser line-width measurement using the self-heterodyne method.

The output of fiber laser can be demodulated with a fiber-optic interferometer as a wavelength discriminator. When the optical path difference of the unbalanced interferometer is large enough, the output intensity noise of the interferometer is caused chiefly by conversion of the laser frequency noise to intensity noise by the interferometer. Therefore the laser intensity noise is negligible [19]. The fluctuations of the laser frequency are measured by using phase-generated carrier method [20]. The 
root-mean square (RMS) of frequency noise spectral density for the laser is shown in Fig. 3. At low frequency $(<400 \mathrm{~Hz})$, the main noise source is environmental fluctuations due to acoustics and vibration. From $400 \mathrm{~Hz}$ to $2 \mathrm{kHz}$, the frequency noise is relatively flat $(<400 \mathrm{~Hz} / \sqrt{ } \mathrm{Hz})$. The finite frequency range of interrogation method limits the measurement of frequency noise for higher frequency. From the measured data in [21-22], it shows $1 / f$ noise below $2 \mathrm{kHz}$. At frequency between a few $\mathrm{kHz}$ to $100 \mathrm{kHz}$, the limiting noise source is thermodynamically fluctuation in the laser cavity [22-23].

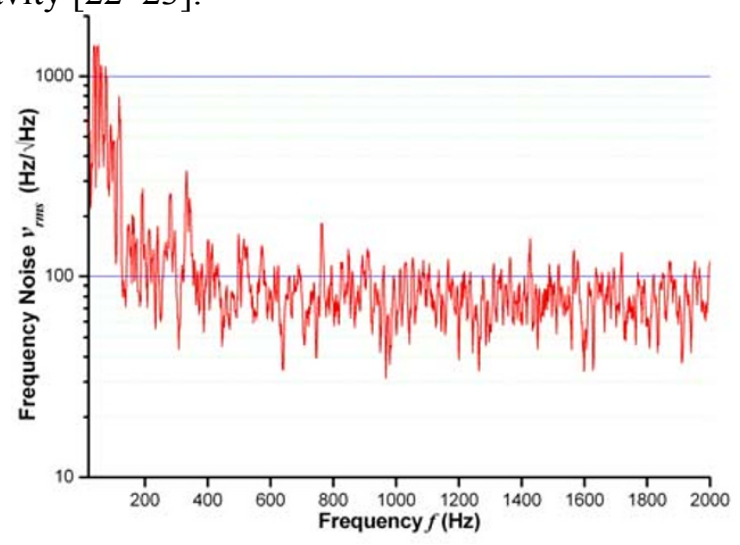

Fig. 3 Measured RMS frequency noise spectral density for DFB fiber laser.

\subsection{Polarization modes of DFB FL}

As mentioned above, DFB structure provides the laser a single longitudinal mode operation. However, most fiber DFB lasers actually operate in two orthogonal polarizations. Single polarization operation is desirable for fiber laser sensor. Many methods are presented to obtain single polarization operation, such as to twist the cavity [24], to stress the phase shift section [25], to introduce a polarization dependent phase shift in the fiber resonator[24], and to employ a polarization dependent feed-back to the cavity [26]. There is a complex model presented for steady state analysis of polarization mode competition in DFB fiber laser by considering the effects of polarization dependent grating non-uniformities, the polarization dependent grating strength, the coupling between the linear polarization states, the spatially and polarization dependent gain hole-burning [27]. A simple analysis based on the relationship between threshold gain coefficient and grating parameters can also provide similar conclusions that reducing the grating strength and slightly deviating from $\pi$ phase shift could contribute to single polarization operation of DFB fiber laser [28]. The polarization modes are measured by using heterodyne method with a single frequency semiconductor as a standard laser source. The heterodyne spectrum is shown in Fig. 4 (a). DFB fiber laser operates in a single polarization mode and single frequency. Further, the polarization behavior of single-polarization DFB fiber laser is investigated by using a polarization analyzer (General Photonics PolaWise). Figure 4(b) shows that the degree of polarization of DFB fiber laser is $99.6 \%$ and no mode hopping is observed.

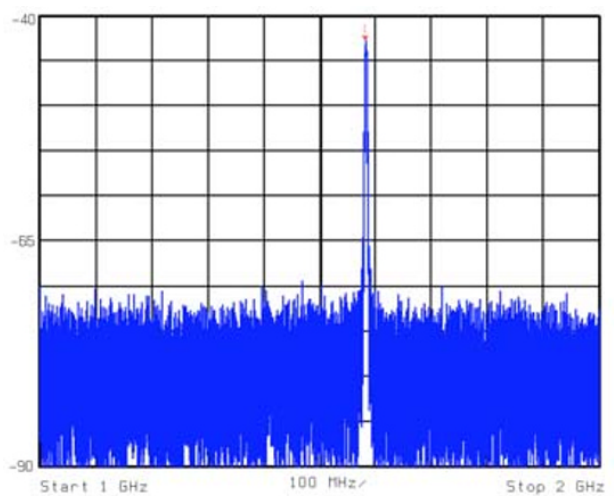

(a)

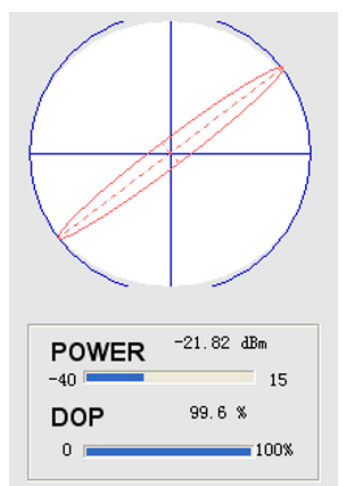

(b)

Fig. 4 (a) Heterodyne detected radio frequency spectrum and (b) poincare sphere representation. 


\section{High-performance wavelength interrogation system}

\subsection{High-resolution wavelength demodulation technique}

The lasing wavelength of DFB fiber laser is determined by the central wavelength in the reflective spectrum of phase-shifted grating, shown as

$$
\lambda_{B}=2 n_{\text {eff }} \Lambda
$$

where $\lambda_{B}$ is the lasing wavelength, $\Lambda$ is the period of grating, and $n_{\text {eff }}$ is the effective reflective index of fiber core. The period $A$ and the effective reflective index $n_{\text {eff }}$ are changed with the environmental conditions, such as strain, temperature, vibration, and acoustic pressure, and hence the lasing wavelength is changed with the measurands. High resolution wavelength demodulation system is a key technique for detection of ultra-weak signals. The traditional wavelength demodulation techniques include: scanning fiberFabry-Perot(FP) filter, matched FBGs, optical spectrum analyzer, and so on. However, the wavelength resolution of traditional demodulation scheme is in the order of picometer or sub-picometer, which is still not enough for some applications.

High resolution wavelength demodulation of DFB fiber laser sensors are based on the interferometric detection[29]. The light from DFB fiber laser passes through an unbalanced fiber interferometer and the interferometric signal $I$ is given by

$$
I=I_{0}\left(1+k \cos \left(\Delta \varphi+\varphi_{0}\right)\right)
$$

where $I_{0}$ is the light intensity of the laser, $k$ is the visibility of interferometric signal, $\Delta \varphi$ is the phase shift of interferometer, and $\varphi_{0}$ is the initial phase. The wavelength shift $\Delta \lambda$ of DFB fiber laser is amplified into the phase shift $\Delta \varphi$ of the interferomtric signal, using an interferometer with an arm-imbalance of $L$.

$$
\Delta \varphi=\frac{2 \pi n L}{\lambda_{B}^{2}} \Delta \lambda_{B} .
$$

Phase demodulation technique is then adopted to extract the phase information, and recover $\Delta \varphi$. The typical phase demodulation scheme includes: heterodyne, passive homodyne using phase generated carrier, homodyne based on a $3 \times 3$ coupler, and so on. The wavelength shift of DFB fiber laser can then be recovered with (4).

When the phase resolution is optimized, a higher wavelength resolution will be obtained in the demodulation system with larger arm-imbalance $L$ of the interferometer. The DFB fiber laser fabricated in our laboratory has a very narrow line-width as $3 \mathrm{kHz}$, the corresponding coherent length can be up to tens of kilometers. The interferometer with large arm-imbalance can then be used, which amplifies the measurands-induced ultra-weak wavelength shift into detectable phase shift. Therefore, high resolution wavelength shift demodulation is accomplished.

Figure 5 shows the schematic of a digital phase-generated carrier (PGC) based wavelength demodulation system for FL sensors[30-31]. The light of DFB FL, which carries the measurands, illuminates the unbalanced Michelson interferometer through an optical isolator. The Michelson interferometer has an arm difference of $10 \mathrm{~m}$, and two Faraday rotation mirrors at its ends, which are used to eliminate the polarization-induced fading[32]. The PGC scheme is used in the system to recover the phase signal and overcome the bias-drift-induced fading [2]. One arm of the interferometer is wrapped onto a piezoelectric (PZT) tube and the phase modulation of the PGC scheme is introduced to the interferometer by electrically modulating the PZT. The PGC frequency is $10 \mathrm{kHz}$ and the magnitude is $2.63 \mathrm{rad}$. The interferometric signals are then received by a photo-detector and an amplifier. The electrical output of the amplifier is digitalized in accordance with the carrier signal using an $\mathrm{A} / \mathrm{D}$ convertor. The PGC phase demodulation is accomplished on a field-programmable gate array (FPGA) board or in a 
personal computer. The PGC algorithm includes: frequency mixing, low pass filtering, differentiation, cross multiplication, subtraction, integration, and high-pass filtering. The digital PGC demodulation scheme has the advantages of high resolution, large dynamic range, easiness of multiplexing, simple configuration, and flexibility.

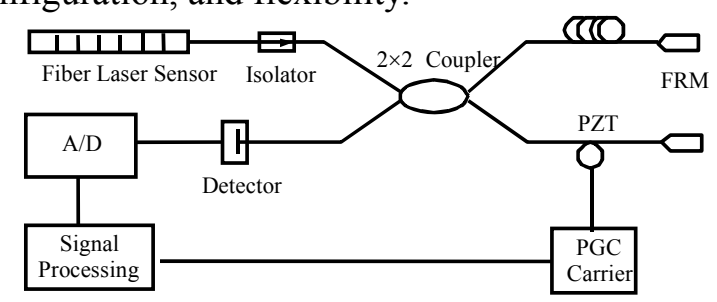

Fig. 5 High resolution wavelength demodulation system.

The wavelength resolution of DFB fiber laser sensor system is limited by system noise level. The key of realizing a high-resolution wavelength demodulation system is the reduction of all kinds of noise in the system, including frequency noise and relative intensity noise (RIN) of DFB fiber laser, electrical circuit noise, and environmental noise on the interferometer. The noise level is measured in a quiet laboratory with the isolation of vibrations and acoustic noise. Figure 6 shows the noise level in the frequency range from $20 \mathrm{~Hz}$ to $2 \mathrm{kHz}$ and it can be observed that the noise level is $3.5 \times 10^{-7} \mathrm{pm} / \sqrt{ } \mathrm{Hz}$ at $1 \mathrm{kHz}$. The relatively high noise level in low frequency range $(20 \mathrm{~Hz}-100 \mathrm{~Hz})$ is probably resulted from two reasons: the frequency noise of DFB fiber laser has a $1 / f$ distribution, and hence it is higher in low frequency; environmental noises (vibrations, acoustics) are mainly in the low frequency region.

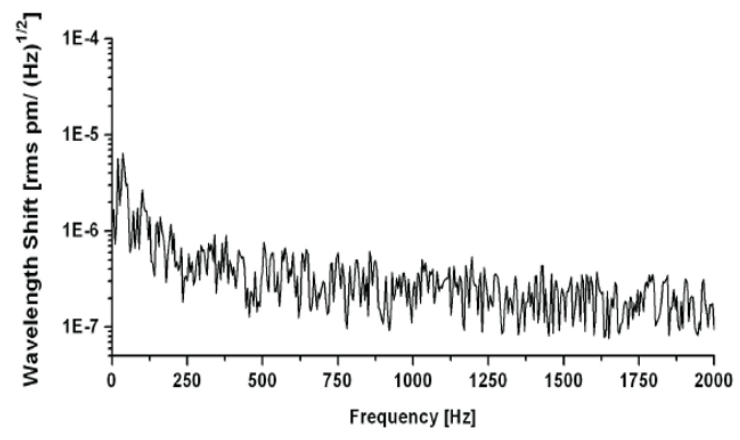

Fig. 6 Noise level of fiber laser sensing system.
When the amplitude of signal is large, the interferometric signal has much more and much higher high frequency components after Bessel expansion. However, the PGC frequency is limited, resulting in spectrum overlapping in the frequency domain, which will introduce serious distortion in the PGC demodulation results [33]. The maximum wavelength shift for a PGC demodulation system is limited. The dynamic range is defined as the ratio of the maximum wavelength shift and the minimum detectable wavelength shift, which is determined by noise level. The dynamic range is shown in Fig. 7, which shows that the maximum system dynamic range of $120 \mathrm{~dB}$ is achieved at $63 \mathrm{~Hz}$. In the lower frequency, the dynamic range is limited by the noise level. In the higher frequency, the dynamic range is declining with the increasing frequency. Signals at the higher frequency are more likely to have spectrum overlap than signals with the same amplitude at low frequency. The maximum wavelength shift is declining with the higher frequency and hence results in the declining system dynamic range with the increasing frequency.

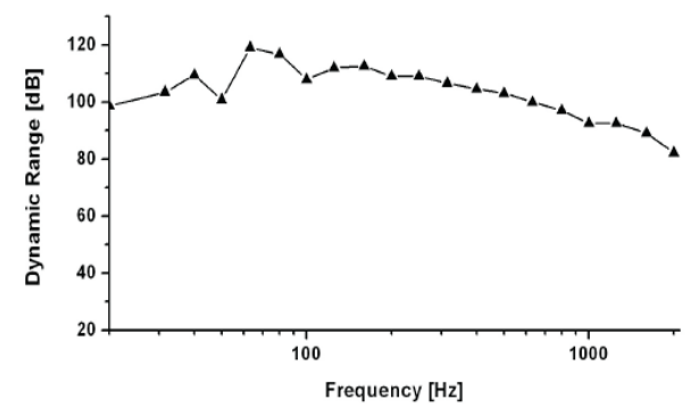

Fig. 7 Dynamic range of fiber laser sensing system.

\subsection{DWDM technique in fiber laser sensing system}

The fiber laser sensors are wavelength encoded, and can easily be multiplexed by dense wavelength division multiplexing (DWDM). Figure 8 shows an 8-channel DFB fiber laser sensor array system based on DWDM. Eight DFB fiber lasers with different lasing wavelengths are serially connected and pumped with a single semiconductor laser. The 
sequence of sensors in the array is arranged according to the threshold power and the slope efficiency of each DFB fiber laser. Optimization is made to equilibrate the lasing power in each sensor element, and it is important for the consistence of each channel in the multi-channel demodulation system. The spectrum of 8-channel DWDM DFB fiber laser sensor array is measured by an optical spectrum analyzer (OSA) and the power equilibrium is within $\pm 5 \mathrm{~dB}$, as shown in Fig. 9. The DWDM optical wavelength signals are converted to phase signals simultaneously and are then separated into each channel by using a thin-film-based de-multiplexing module. Photoelectric detection, amplification, analog-to-digital (A/D) convert, and PGC demodulation are all accomplished in each individual channel.

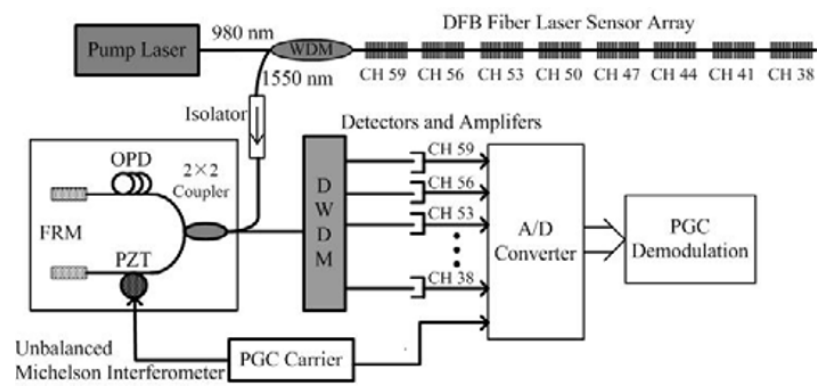

Fig. 8 8-channel fiber laser sensor network based on DWDM.

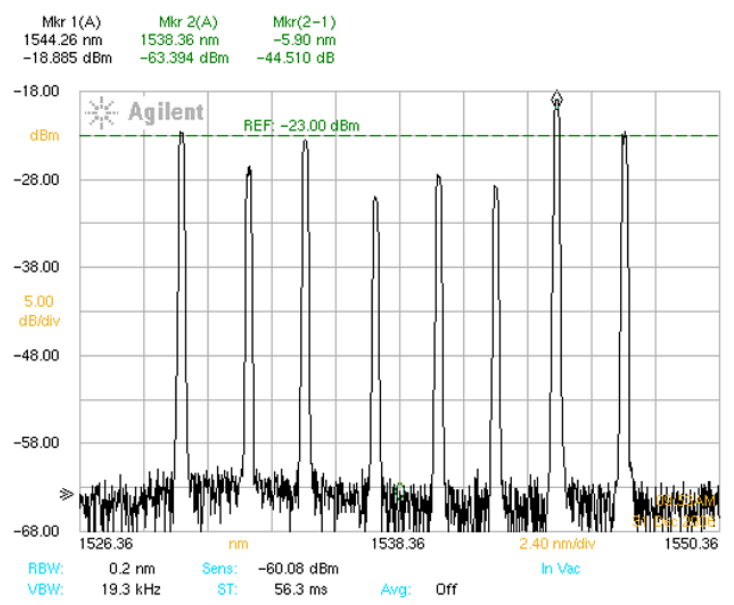

Fig. 9 Optical spectrum of 8-channel DFB fiber laser array.

The crosstalk in adjacent channels is an important problem. A detailed study on analyzing the crosstalk in a wavelength division multiplexed fiber laser sensor array system based on a digital phase generated carrier interferometric interrogation scheme is carried out. The crosstalk effects are mainly induced by the limited optical channel isolation of a DWDM. In order to analyze the signal crosstalk induced by channel isolation and find the necessary channel isolation to keep crosstalk negligible, we proposed a novel calculation method via Bessel function expansion of the output signal [34]. The mathematical model of the PGC demodulation of a two-channel fiber laser sensors are built up. The frequency components of output signals in each channel are obtained by Bessel function expansion. The crosstalk can be negligible when all the frequency components related with the crosstalk are suppressed below noise level. Therefore, we can calculate the required channel isolation value of DWDM by the following simple criterion:

$$
m<1.87 \varphi_{\text {noise }}
$$

where $m$ is the light intensity ratio of crosstalk channel to another channel, $\varphi_{\text {noise }}$ is the interferometer phase noise.

With the measured system phase noise applied on (5), the maximal value of $m$ could be calculated to about $7.48 \times 10^{-5}$, which corresponds to $41.3 \mathrm{~dB}$ of the necessary DWDM channel isolation. In order to demonstrate the crosstalk analysis result, we built up a two-element serial fiber laser sensor system, as shown in Fig. 10. The configuration was similar to Fig. 5, except that there was no DWDM at the output of fiber interferometer, and a tunable fiber attenuator was inserted between two laser sensors. Two lasers were chosen to operate at $1534.996 \mathrm{~nm}$ and $1532.620 \mathrm{~nm}$. The return light was split to two branches via a $2 \times 2$ coupler, and an OSA (Agilent 86140) was employed to measure the light intensity ratio. We mounted the laser cavities on two PZT stretchers respectively. Two test signals with different frequencies and amplitudes were applied on the PZT stretchers. The different light intensity ratios of fiber laser sensor(FLS) $\lambda_{1}$ and FLS $\lambda_{2}$ could be achieved by adjusting the tunable fiber attenuator, 
which was similar to adjusting the channel isolation of DWDM in a multiplexed fiber laser system. At the same time, we observed the output of the demodulator. Thus, we could determine the minimal required intensity ratio of two lasers by adjusting the attenuator, until the intensity of FLS $\lambda_{2}$ was poor enough that the corresponding output signal could not be observed even though the test signal on FLS $\lambda_{2}$ was very large. The experimental results have shown that the crosstalk from FLS $\lambda_{2}$ to FLS $\lambda_{1}$ is reduced with the increase of light intensity ratio of two lasers. When the ratio reaches about $41.9 \mathrm{~dB}$, the crosstalk is covered by the system noise and cannot be observed. This experimental result is nearly consistent with above mentioned calculations about necessary channel isolation of a DWDM. According to the preceding conclusion, we finally adopted an 8-channel DWDM module with a $300 \mathrm{GHz}(2.4 \mathrm{~nm})$ channel interval and a $50 \mathrm{~dB}$ channel isolation in our 8-channel fiber laser array system, as shown in Fig. 11. Experimental results demonstrated that there was no measurable crosstalk between the output channels.

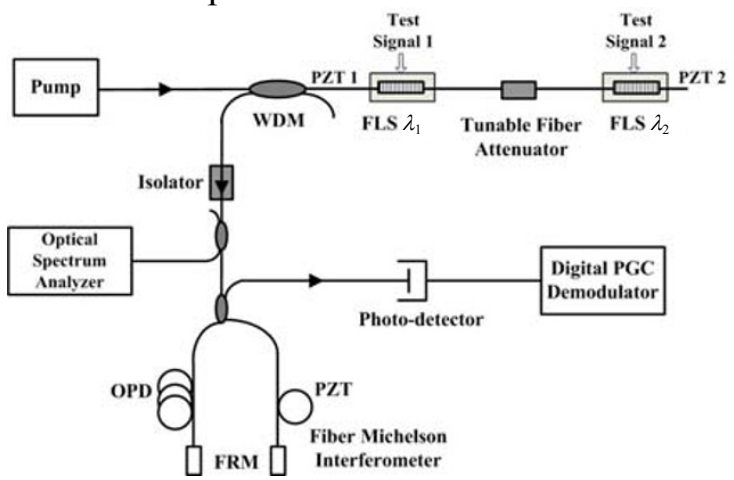

Fig. 10 Experiment of the crosstalk analysis of two DFB fiber laser sensors.

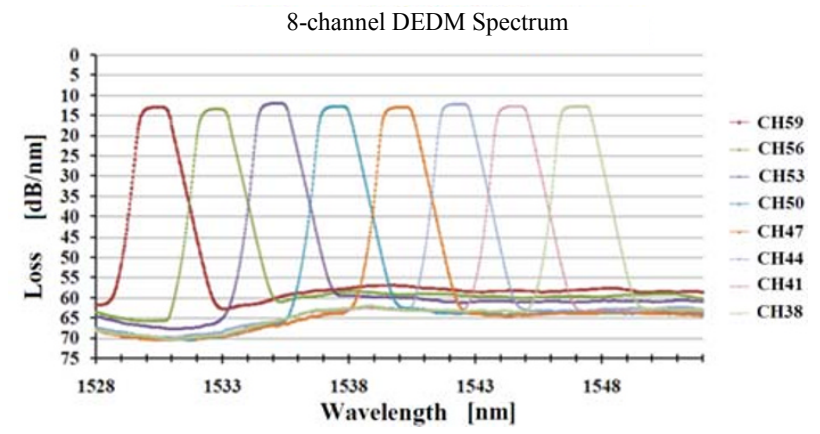

Fig. 11 8-channel DWDM spectrum.
When the fiber laser sensor network is used in the applications such as underwater sonar system or seismic signal acquisition, data of multi-channel are needed in the analysis of intensity and the position of object. Phase consistency between different channels of fiber laser sensor system has great effect on beam-forming and target locating in the detection of underwater acoustics and seismic wave. So it is important to study the phase consistency between different channels in the DWDM fiber laser system array system. We divide the system into analog part and digital part, and analyze them individually. The phase delay in the analog part includes the phase difference of the transmission of laser in different wavelengths, photoelectric convertings, amplifiers, and $\mathrm{A} / \mathrm{D}$ convertors; the phase difference in the digital part is induced by low-pass-filtering, differentiating, integrating, and high-pass-filtering in the PGC algorithm. Theoretical analysis, corresponding calculations, and experiments show that the influence of laser wavelength difference can be neglected, and low-pass-filter is the main factor affecting the phase consistency. By using low-pass-filter with the same type, configuration, length, and order in each DWDM PGC channel, the phase consistency can be optimized to be within $1^{\circ}$ in the DWDM fiber laser sensor network.

\section{Design and applications of FL sensor and sensor array}

\subsection{FL Hydrophone and towed array}

The first laboratory demonstration of an ultra-thin fiber laser hydrophone (FLH) array was reported by Hill et al. in 1999[35], with three fiber lasers on a single fiber. Subsequently, there was a number of reports about fiber laser hydrophones, with configurations mostly based on polymer coating on bare fiber laser[4, 35-36], or shielded polymer coating on fiber laser[37].

Fiber laser hydrophones are likely to work better if the sensitivity is enhanced while the frequency 
response is flattened. We proposed an ultrathin fiber laser hydrophone with enhanced sensitivity by using a thin metal cylinder and a piston-like diaphragm, as shown in Fig. 12 [38]. The sensor is covered by rubber coating. Inside the rubber coating is sensor shell which is made of a thin-wall metal cylinder with many sensing holes on its surface. The rubber is made of sound transmission rubber. The transmission rate of this type of polyurethane rubber is higher than $99 \%$. The sensor shell is filled with oil to transmit acoustic pressure. So acoustic pressure can couple inside the sensor shell through the sensing holes and act on the surface of piston-like diaphragms. The diaphragm, which is made of rubber, is pressurized in axial direction, inducing an axial tension strain in bare fiber laser. A hard core, which is made of copper, is buried at the center of each diaphragm when the diaphragm is cast to enhance the sensitivity and to fix the fiber.

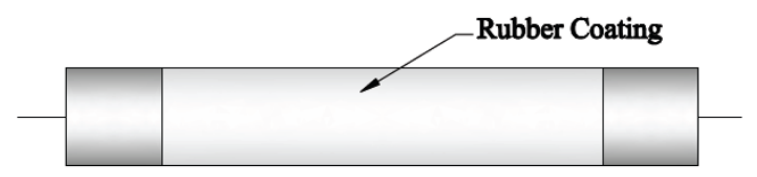

(a)

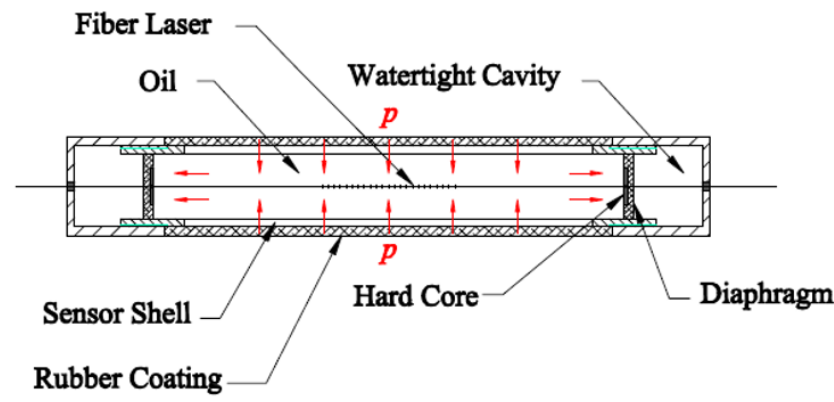

(b)

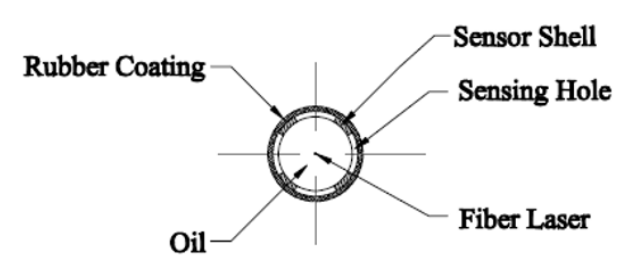

(c)

Fig. 12 Schematic of the fiber laser hydrophone: (a) outside view, (b) section view, and (c) cross-section view.

The sensitivity of the hydrophone is given by

$$
\frac{\Delta \lambda_{B}}{p}=\frac{\left(1-p_{e}\right) \frac{R^{4}}{64 D}\left(1-\left(\frac{r}{R}\right)^{4}+4\left(\frac{r}{R}\right)^{2} \ln \frac{r}{R}\right)}{\frac{L}{2}+\frac{A E_{f} R^{2}}{16 \pi D}\left[1-\left(\frac{r}{R}\right)^{2} \frac{1-\left(\frac{r}{R}\right)^{2}+4 \ln ^{2} \frac{r}{R}}{1-\left(\frac{r}{R}\right)^{2}}\right]}
$$

where $p_{\mathrm{e}}$ is the effective photoelastic constant of fiber, $R$ is the radius of the diaphragm, $t$ is the thickness of the diaphragm, $r$ is the radius of the hard core, $E$ is the Young's modulus of the diaphragm, $A$ is the cross section area of the fiber, $E_{f}$ is the Young's modulus of the fiber, $D$ is the bending strength of the diaphram.

DFB fiber lasers are used as the sensing element of FLH. The cavity length of DFB laser used in the hydrophone is $36 \mathrm{~mm}$ and the reflectivity of the grating is $99 \%$. The center wavelength of DFB fiber laser is about $1535 \mathrm{~nm}$. Theoretical analysis shows that the Young's modulus of the diaphragm and the radius of the hard core have significant effect on pressure sensitivity. By optimizing these two parameters, a pressure sensitivity of $7 \mathrm{~nm} / \mathrm{MPa}$ has been achieved when the radius of the hard core is $1.2 \mathrm{~mm}$. The hydrostatic test results agree well with theoretical analysis and measurements on frequency response indicate that as the Young's modulus of the diaphragm increases, the frequency response will get flatter.

When FLH operates in deep water, the wavelength of fiber laser will shift a lot due to hydrostatic pressure, which will induce severe problems when using DWDM technique[39]. Hence, pressure gradient structure is introduced in enhancing the sensitivity of FLH and compensating the hydrostatic pressure[40]. Owing to the orifice at the sensor shell, the water can enter into the sensor and the hydrostatic pressure is compensated while an ultra-high sensitivity has been achieved.

The development of fiber laser hydrophone with reduced size has enabled the building of ultra-thin arrays of wavelength multiplexed acoustic sensors. Thus, a towed array comprising eight fiber 
hydrophones multiplexed onto a single fiber has been constructed with a diameter of $30 \mathrm{~mm}$, as illustrated in Fig. 13.

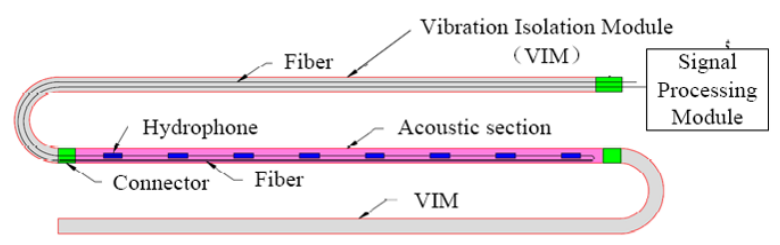

Fig. 13 Fiber laser hydrophone towed array.

\subsection{FL sensors in seismic sensing}

A better known exploitation of fiber-optic sensing technology has been in the development of seismic sensing arrays[41-44]. We have developed a lightweight, highly sensitive fiber laser geophone (FLG) for underwater seismic measurements[45]. The proposed FLG is shown in Figs. 14 and 15. Two brass diaphragms, as the sensing element, are installed in the sensor shell which is made of a thin-wall metal cylinder. A mass is installed between two diaphragms to induce larger deformation of the diaphragms due to acceleration. The DFB fiber laser is anchored at the sensor shell and the mass will be elongated or shortened by the seismic wave. There are two holes at the side of sensor shell. Water can fill the geophone from the two holes, which can compensate the static pressure in the water.

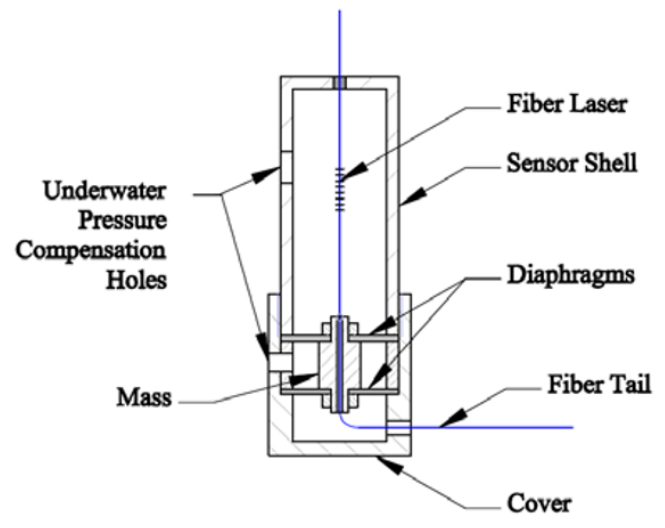

Fig. 14 Schematic of the FL geophone.

The sensitivity of FLG is tested to be $25 \mathrm{pm} / \mathrm{g}$, as shown in Fig. 15. Due to its low noise floor of $10^{-5} \mathrm{pm} / \sqrt{ } \mathrm{Hz}$ at $100 \mathrm{~Hz}$, a minimum detectable signal of $0.4 \mathrm{mg}$ is achieved. Field test shows that FLG array has a good consistency, which implies the proposed FLG is promising in the application to oilfield exploration.

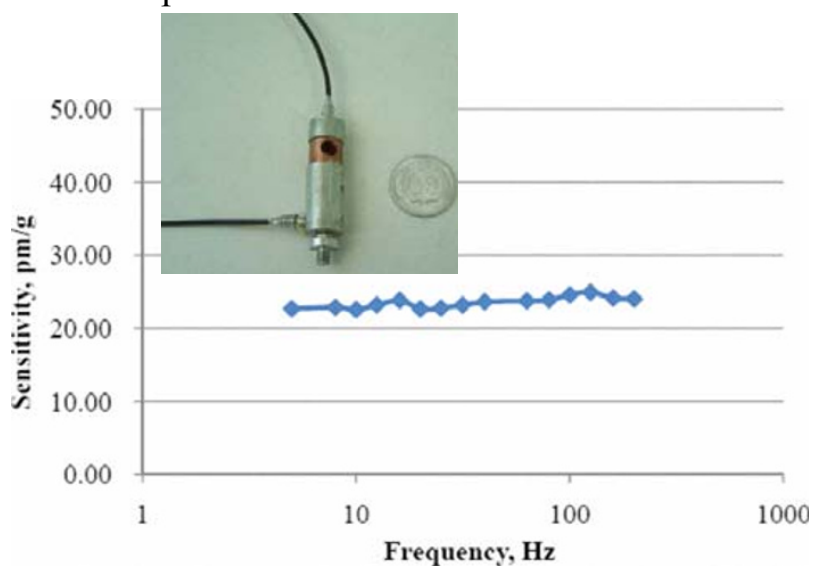

Fig. 15 Photo of the geophone and the tested sensitivity.

A 4-element FLH array is also demonstrated for the detection of seismic signals. The hydrophone array is installed in a well with $20 \mathrm{~cm}$ apart. Seismic signals are stimulated by a shock of free falling from $1 \mathrm{~m}$ high and $10 \mathrm{~m}$ far away from the well. The received signal is compared with that of a 2-element traditional piezoelectric (PZT) hydrophone array, as shown in Fig. 16. The results show that hydrophones have better performance over traditional PZT hydrophones.

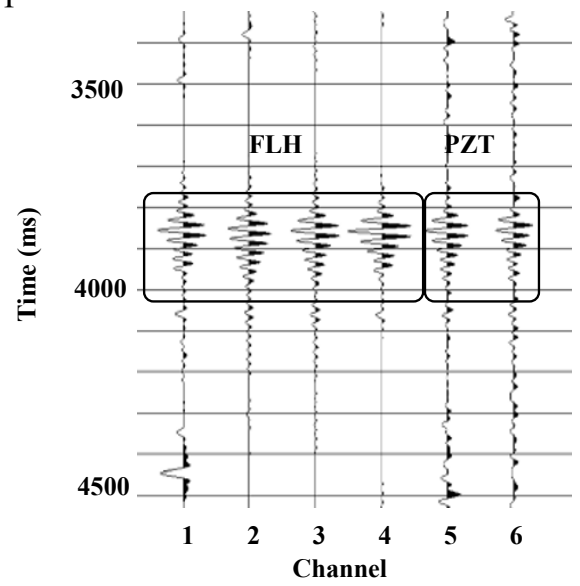

Fig. 16 Seismic signals received by hydrophone array.

\section{Conclusions}

Narrow line-width, low phase nose, single polarization mode DFB fiber laser can be achieved 
by optimizing the design and fabrication process. Utilizing high resolution wavelength demodulation technique and DWDM technique, high performance fiber laser sensing system is demonstrated. Field test shows that the fiber laser sensing system is promising in the application to towed array and oilfield exploration.

\section{Acknowledgement}

The author gives thanks to the support by National Science Foundation China under Grant No. 61077059 and 41074128, 863 Program of China under Grant No. 2009AA11Z212, and the Knowledge Innovation Program of the Chinese Academy of Sciences under Grant No. ISCAS2008T05.

\section{References}

[1] K. T. V. Grattan and D. T. Sun, "Fiber optic sensor technology: an overview," Sensor and actuators A-phys, vol. 82, no. 1-3, pp. 40-61, 2000.

[2] C. K. Kirkendall and A. Dandridge, "Overview of high performance fibre-optic sensing," J. Phys. D, vol. 37, no. 18, pp. R197-R216, 2004.

[3] G. A. Cranch, G. M. H Flockhart, and C. K. Kirkendall, "Distributed feedback fiber laser strain sensors," IEEE Sensors J., vol. 8, no. 7, pp. 1161-1172, 2008.

[4] B. H. David J. Hill, Jolyon De Freitas, Sean D. Thomas, and Louise Hickey, "DFB fibre-laser sensor developments," presented at 17th International Conference on Optical Fibre Sensors, Brugge, Belgium, 2005.

[5] S. Foster, A. Tikhomirov, M. Englund, H. Inglis, G. Edvell, and M. Milnes, "A 16 Channel Fibre Laser Sensor Array," presented at ACOFT \& AOS Conference 2006, Melbourne, VIC, Australia, July 10-13, 2006.

[6] A. D. Kersey, K. P. Koo, and M. A. Davis, "Fiber Optic Bragg Grating Laser Sensors," presented at Conference on Fiber Optic and Laser Sensors XII, San Diego, CA, 1994.

[7] J. L. Zyskind, V. Mizrahi, D. J. Digiovani, and J. W. Sulhoff, "Short single frequency erbium-doped fibre laser," Electron. Lett., vol. 28, no. 15, pp. 1385-1387, 1992.

[8] Y. O. Barmenkov, D. Zalvidea, S. Torres-Peiro, J. L. Cruz, and M.V. Andres, "Effective length of short Fabry-Perot cavity formed by uniform fiber Bragg gratings," Opt. Express, vol. 14, no. 14, pp.
6394-6399, 2006.

[9] V. C. Lauridsen, J. H. Povlsen, and P. Varming, "Design of DFB fibre lasers," Electron. Lett., vol. 34, no. 21, pp. 2028-2030, 1998.

[10] W. H. Loh and R. I. Laming, " $1.55 \mu \mathrm{m}$ phase-shifted distributed feedback fiber laser," Electron. Lett., vol. 31, no. 17, pp. 1440-1442, 1995.

[11] E. Ronnekleiv, S. W. Lovseth, and J. L. Kringleboth, "Er-doped fiber distributed feedback lasers properties, applications and design considerations," in Conference on Fiber-Based Component Fabrication, Testing, and Connectorization, Brugge, Belgium, pp. 69-80, 2002.

[12] M. Yamada and K. Sakuda, "Analysis of almost-periodic distributed feedback slab waveguides via a fundamental matrix approach," Appl. Optics, vol. 26, no. 16, pp. 3474-3478, 1987.

[13] K. O. Hill and G. Meltz, "Fiber Bragg grating technology fundamentals and overview," J. Lightw. Technol., vol. 15, no. 8, pp. 1263-1276, 1997.

[14] I. Bennion, J. A. R. Williams, L. Zhang, K. Sugden, and N. J. Doran, "UV-written in-fibre Bragg gratings," Opt. Quant. Electron., vol. 28, no. 2, pp. 93-135, 1996.

[15] J. Canning and M. G. Sceats, "Pi-phase-shifted periodic distributed structures in optical fibers by UV post-processing," Electron. Lett., vol. 30, no. 16, pp. 1344-1345, 1994.

[16] V. C. Lauridsen, J. H. Povlsen, and P. Varming, "Optimising erbium-doped DFB fibre laser length with respect to maximum output power," Electron. Lett., vol. 35, no. 4, pp. 300-302, 1999.

[17] K. Yelen, M. N. Zervas, and L. M. B. Hickey, "Fiber DFB laser with ultimate efficiency," J. Lightw. Technol., vol. 23, no. 1, pp. 32-43, 2005.

[18] K. M. Abramski, "Laser diode linewidth measurements," presented at the Transparent Optical Networks, Proceedings of 2003 5th International Conference on Transparent Optical Networks, June 29-July 3, 2003.

[19] Z. Meng, Y. M. Hu, S. D. Xiong, G. Stewart, G. Whitenett, and B. Culshaw, "Phase noise characteristics of a diode-pumped Nd : YAG laser in an unbalanced fiber-optic interferometer," Appl. Optics, vol. 44, no. 17, pp. 3425-3428, 2005.

[20] H. Xiao, F. Li, and Y. Liu, "Crosstalk analysis of a fiber laser sensor array system based on digital phase-generated carrier scheme," J. Lightw. Technol., vol. 26, no. 10, pp. 1249-1255, 2008.

[21] E. Ronnekleiv, "Frequency and intensity noise of single frequency fiber Bragg grating lasers," Opt. Fiber Technol., vol. 7, no. 3, pp. 206-235, 2001.

[22] S. Foster, A. Tikhomirov, and M. Milnes, "Fundamental thermal noise in distributed feedback fiber lasers," IEEE J. Quantum Elect., vol. 43, no. 
5-6, pp. 378-384, 2007.

[23] K. H. Wanser, "Fundamental phase noise limit in optical fibers due to temperature fluctuations," Electron. Lett., vol. 28, no. 1, pp. 53-54, 1992.

[24] Z. E. Harutjunian, W. H. Loh, R. I. Laming, and D. N. Payne, "Single polarisation twisted distributed feedback fibre laser," Electron. Lett., vol. 32, no. 4, pp. 346-348, 1996.

[25] W. Fan, B. Chen, X. C. Li, L. R. Chen, and Z. Q. Lin, "Stress-induced single polarization DFB fiber lasers," Opt. Commun., vol. 204, no. 1-6, pp. 157-161, 2002.

[26] S. Yamashita and G. J. Cowle, "Single-polarization operation of fiber distributed feedback (DFB) lasers by injection locking," J. of Lightw. Technol., vol. 17, no. 3, pp. 509-513, 1999.

[27] E. Ronnekleiv, M. N. Zervas, and J. T. Kringlebotn, "Modeling of polarization-mode competition in fiber DFB lasers," IEEE J. Quant. Elect., vol. 34, no. 9, pp. 1559-1569, 1998.

[28] T. Xu, F. Li, Y. Liu, and Lihui Liu, "Characteristic mode analysis of distributed feedback fiber lasers," Chin. J. Lasers, vol. 34, no. 10, pp. 1358-1361, 2007(in Chinese).

[29] H. Xiao, F. Li, and Y. Liu, "Recent advance on technique of high resolution wavelength shift demodulation," Laser \& Optoelectronics Progress, vol. 44, no. 4, pp. 6, 2007 (in Chinese).

[30] H. Xiao, Y. Wang, L. Liu, and Y. Liu, "High-resolution fiber laser sensor system," Chin. J. Lasers, vol. 35, no. 1, pp. 5, 2008(in Chinese).

[31] J. He, T. Xu, Y. Wang, and Y. Liu, "High performance distributed feedback fiber laser sensor array system," presented at 2009 Asia Communications and Photonics conference and Exhibition, Shanghai, Nov. 2-6, 2009.

[32] L. Qi, H. Xiao, F. Li, and Y. Liu, "Study of polarization-insensitive fiber optic interferometers for wavelength demodulation," Laser \& Infrared, vol. 38, no. 3, pp. 4, 2008(in Chinese).

[33] Y. Hu, M. Ni, and Z. Meng, "Dynamic Range of Fiber Optic Hydrophone Using Digitized Phase Generated Carrier," Laser \& Optoelectronics Progress, vol. 42, no. 2, pp. 5, 2005(in Chinese).

[34] H. Xiao, F. Li, and Y. Liu, "Crosstalk Analysis of a Fiber Laser Sensor Array System Based on Digital Phase-Generated Carrier Scheme," J. Lightw. Technol., vol. 26, no. 10, pp. 1249-1355, 2008.

[35] P. Nash, D. J. Hill, D. A. Jackson, D. J. Web, S. F. O'Neill, I. Bennion, and L. Zhang, "A fiber laser hydrophone array," presented at the SPIE
Conference on Fiber Optic Sensor Technology and Applications, Boston, 1999.

[36] P. E. Bagnoli, N. Beverini, R. Falciai, E. Maccioni, M. Morganti, F. Sorrentino, F. Stefani, and C. Trono, "Development of an erbium-doped fibre laser as a deep-sea hydrophone," J. Opt. A, vol. 8, no. 7, pp. S535-539, 2006.

[37] L. V. Hansen and F. Kullander, "Modelling of hydrophone based on a DFB fiber laser," presented at the XXI International Congress of Theoretical and Applied Mechanics, Warsaw, Poland, August 15-21, 2004.

[38] W. Zhang, F. Li, and H. Xiao, "Fiber Laser Hydrophone Based on DoubleDiaphragms: Theory and Experiment," J. Lightw. Technol., vol. 26, no. 10, pp.1349-1342, 2008.

[39] S. Goodman, A. Tikhomirov, and S. Foster, "Pressure Compensated Distributed Feedback Fibre Laser Hydrophone," presented at 19th International Conference on Optical Fibre Sensors, Perth, Western Australia, April 14-18, 2008.

[40] W. Zhang, F. Zhang, F. Li, and Y. liu, "Investigation on a pressure-gradient fiber laser hydrophone," Meas. Sci. Technol., vol. 21, no. 9, pp. 094037-094041, 2010.

[41] S. Knudsen, G. B. H avsgárd, A. Berg, H. Nakstad, and T. Bostick, "Permanently Installed High Resolution Fiber-Optic 3C/4D Seismic Sensor Systems for In-Well Imaging and Monitoring Applications," in Proc. of SPIE(Sixth Pacific Northwest Fiber Optic Sensor Workshop, Bellingham, WA), vol. 5278, pp. 51-55, 2003.

[42] Y. Zhang, S. Li, R. Pastore, Z. Yin, and H. Cui, "Detection of seismic signal using fiber Bragg grating sensors," presented at Conference on Photonic Sensing Technologies, Boston, MA, Oct. 1-4, 2006.

[43]A.V. Strudley and P. J. Nash, "Efficient Optical Architectures for Permanent Seismic Reservoir Monitoring Arrays," presented at the EAGE 69th Conference \& Exhibition, London, UK, June 11-14, 2007.

[44] P. Nash, A. Nash, R. Crickmore, and J. DeFreitas, "High Efficiency TDM/WDM Architectures for Seismic Reservoir Monitoring," presented at 20th International Conference on Optical Fibre Sensors, Edinburgh, UK, 2009.

[45] W. Zhang, X. Li, F. Zhang, F. li, and Y. Liu, "Underwater fiber laser geophone: theory and experiment," presented at 2009 Asia Communications and Photonics conference and Exhibition, Shanghai, Nov. 2-6, 2009. 